\title{
A Clinical Study of Impact of HIV Infection and HAART on Serum Lipids in People Living in Sikkim
}

\author{
Aroop Mohanty ${ }^{1 *}$, Manish Pandey ${ }^{2}$, A.P. Mohanty ${ }^{2}$ and Pratima Gupta ${ }^{1}$ \\ ${ }^{1}$ Department of Clinical Microbiology, AIIMS Rishikesh, India \\ ${ }^{2}$ Department of Medicine, KIIMS Bhubaneswar, India \\ *Corresponding author
}

\begin{tabular}{|l|}
\hline Key w or d s \\
HAART, ART, \\
Cholesterol, HIV \\
\hline Article Info \\
\hline $\begin{array}{l}\text { Accepted: } \\
\text { 04 September } 2018 \\
\text { Available Online: } \\
\text { 10 October } 2018\end{array}$ \\
\hline
\end{tabular}

A B S T R A C T

The advent of HAART has been associated with a profound reduction in morbidity and mortality from HIV/AIDS. However, side effects and toxicities associated with HAART may lead to an increased risk for cardiovascular diseases. The aim of this study was to determine the prevalence of dyslipidemia and determining factors of derangements in lipid profile associated with use of HAART regimens in people living with HIV/AIDS. This cross-sectional study was carried out from October 2012 to April 2014. Patients aged above 18 years, who were newly started on first line antiretroviral therapy were included in this study. Lipid profile was determined after overnight fast and dyslipidemia was diagnosed according to US National Cholesterol Education Program criteria. Sociodemographic characteristics were also collected using a questionnaire. Data was analyzed using STATA; chi-square test, t-test, ANOVA and logistic regression were computed. Significant change in LDL level was noted in patients on follow up. Trend of increasing T.C. level was noted on follow up among patients on ART. Patient on ZLN ART regimen had significant changes in HDL, LDL, TC, and TG favoring dyslipidemia. Similar changes were also noted in patients on ZLE. It was observed that patients having trend towards dyslipidemia also had improving CD4 counts. We can conclude that dyslipidemia among HIV-infected patients using HAART are significantly associated with longer duration of treatment. Lipid profile and other cardiovascular risk factors should be monitored in patients on ART so that any negative effects of HAART can be optimally managed.

\section{Introduction}

HIV is one of the leading causes for burden of diseases worldwide. The advent and widespread use of combination antiretroviral therapy (ART) referred to as highly active antiretroviral therapy (HAART) in the mid 1990's, has led to a dramatic decline in the incidence of immunodeficiency-related events, including causes of death (Grinsztejn et al., 2013). Suppression of HIV replication is an important component in prolonging life as well as in improving the quality of life of many. Adequate suppression requires strict adherence to prescribed regimens of ARV drugs. This has been facilitated by the conformations of ARVs and the development of once daily regimens. Despite these 
spectacular results, a lot of queries remain and a lot of issues are under debate. Almost all antiretroviral drugs have side effects of varying severity. Metabolic complications of chronic use of highly active retroviral therapy include diabetes mellitus, insulin resistance and dyslipidemia. It leads to an increased risk for cardiovascular morbidity and mortality in HIV infected individuals (Grover et al., 2005). HIV related lipodystrophy, is a condition with an elevation of plasma triglycerides, total cholesterol and sugar levels. The characteristics of dyslipidemia include elevated levels of total cholesterol (TC), LDLcholesterol (LDL-c), triglycerides (TG), decreased HDL-cholesterol (HDL-c) and increased triglyceride levels (Barbaro, 2006). There is limited information on dyslipidemia prevalence in HIV-infected patients receiving HAART worldwide, especially in our country. The prevalence of dyslipidemia in resource limited $s$ in resource-limited settings like India has not been well studied. Current WHO antiretroviral guidelines do not recommend that lipid monitoring should be conducted in patients receiving first- line HAART (Biron $e t$ al., AIDS, 2012). The main objective of this present study was to determine the prevalence of dyslipidemia and determining factors of derangements in lipid profile associated with the use of HAART regimens in people living with HIV infection in the East Sikkim region of India.

\section{Materials and Methods}

\section{Study settings and participants}

This was a hospital- based cross-sectional study. It was carried out in the HIV center of a tertiary care teaching hospital from October 2012 to April 2014. Patients aged above 18 years, who were newly started on first line antiretroviral therapy were included in this study. Participants receiving lipid altering therapies, pregnant women, known diabetes mellitus patients, those with renal failure were excluded.

\section{Data collection and measurements}

Informed consent was taken from each patient. Structured questionnaires were used to collect data on the socio-demographic characteristics and patient's records were reviewed for information on their current CD4 T cell count, HIV and HAART status. Body mass index and blood pressure were also measured. Waist circumference was measured using a nonelastic measuring tape at the highest level of iliac crest with the patient's feet 1 foot apart. The patients were also enquired about history of alcohol intake and any form of physical exercise. About $5 \mathrm{ml}$ of venous blood sample was collected from each participant through venipuncture after a $12 \mathrm{~h}$ overnight fast and centrifuged at 2500 cycles/min for $10 \mathrm{~min}$, and serum was obtained for fasting blood sugar and lipid profile measurement. Baseline investigations were carried out and tabulated for all the patients. All the patients who were given the three drug regimen were periodically monitored at 03 months and 06 months. They were also screened for opportunistic infections during each visit.

\section{Results and Discussion}

\section{Demographic data}

A total of 112 patients were enrolled in the study out of which 55 patients were excluded. Remaining 57 patients who fulfilled the inclusion and exclusion criteria were studied out of which 51 (89.5) were males and remaining 6 (10.5) were females. Maximum number of patients was in the age group of 3039 comprising a total of $22(38.5 \%)$. ART profile:

The patients were started on combination ART based on NACO guidelines and no 
experimental single drug regimen was given. The most common regimen was ZLN on which there were 30 patients (57\%), 19 patients $(33 \%)$ were on ZLE, 6 patients $(10.5 \%)$ were on SLE and 1 each in ZNS and SNL regimen. Triceps skin fold thickness (TSFT):

All patients were followed up with TSFT as a marker for obesity and dyslipidemia. It was measured by skin calipers.

At initiation of ART 22 patients $(38.5 \%)$ had TSFT between 5-10 $\mathrm{mm}$ and 33 patients (57.8\%) between $10-22$. Six months later the distribution changed to $24(42.1 \%)$ and 30 $(52.6 \%)$ respectively.

\section{HDL-C profile}

Patients were followed up with lipid profile in the form of HDL, LDL, TC and TG at the baseline, 3 months and 6 months. At baseline all the 57 patients had HDL below 50mg/dl while at 6 months majority (34/57) were below 40mg/dlas shown in table ( $\mathrm{p}$ value $0.0001)$. The incidence of dyslipidemia was 965 per 1000 per 6 months.

\section{TC profile}

At baseline all patients had TC below 200 $\mathrm{mg} / \mathrm{dl}$. This distribution changed at 3 months where about $35 \%$ patients measured above $200 \mathrm{mg} / \mathrm{dl}$ whereas at 6 months more than half of the patients had levels more than $240 \mathrm{mg} / \mathrm{dl}$ as shown in the table ( $\mathrm{p}$ value 0.0001 ). The incidence of dyslipidemia was 578.9 per 1000 per 6 month.

\section{TG profile}

At baseline all patients measured TG below $150 \mathrm{mg} / \mathrm{dl}$. This also changed at 6 months where about $42 \%$ of patients had TG more than $200 \mathrm{mg} / \mathrm{dl}$. The incidence of dyslipidemia was 649.1 per 1000 per 6 months.

\section{LDL profile}

Similarly the LDL levels were below $100 \mathrm{mg} / \mathrm{dl}$ for all 57 patients at baseline which showed significant changes at 6 months. $15 \%$ of the patients had LDL levels more than $190 \mathrm{mg} / \mathrm{dl}$ after taking treatment for 6 months. The incidence of dyslipidemia was 508.7 per1000 per 6 months.

\section{HDL-C Profile}

\begin{tabular}{|l|l|l|l|}
\hline HDL $(\mathrm{mg} / \mathrm{dl})$ & Baseline & $\mathbf{3}$ months & $\mathbf{6}$ months \\
\hline$>\mathbf{5 0}$ & 57 & 6 & 2 \\
\hline $\mathbf{4 0 - 4 9}$ & 0 & 29 & 21 \\
\hline$<40$ & 0 & 22 & 34 \\
\hline
\end{tabular}

Highly active antiretroviral therapy (HAART) has had a significant impact on the natural history of HIV infection, leading to a remarkable decrease in its morbidity and mortality, but is frequently associated with clinical and metabolic complications. Fat redistribution or lipodystrophy, hypertriglyceridemia, hypercholesterolemia, insulin resistance and diabetes mellitus have been extensively reported in subjects treated with protease inhibitor based anti-retroviral agents. In particular, dyslipidemia occurs in up to $70-80 \%$ of HIV-infected individuals receiving HAART and can be associated with all the available PIs, although hypertriglyceridemia appears to be more frequent in patients treated with ritonavir, saquinavir and lopinavir. The potential long term consequences of HAART-associated hyperlipidemia are not completely 
understood, but an increased risk of premature coronary artery disease has been reported in young HIV patients receiving PIs. Dietary changes, regular aerobic exercise and switching to a PI-sparing regimen may act favorably on dyslipidemia. Lipid lowering therapy is often required with statins or fibrates. The choice of hypolipidaemic drugs should take into account potential pharmacological interactions with antiretroviral agents. Before the availability of highly active antiretroviral, studies in persons infected with HIV type 1 demonstrated lipid abnormalities. Men with HIV infection were reported to have hypercholesterolemia with and without hypertriglyceridemia. An association between plasma triglyceride levels and circulating interferon levels has been observed in persons with AIDA; however, the mechanism for hypercholesterolemia in HIV and other infections is not known. A pattern of hyperlipidemia has been observed in patients treated with protease inhibitors. Several other metabolic abnormalities have been observed in HAART recipients. Anecdotal reports of premature atherosclerosis in HAART recipients and the observed at hero genic plasma lipid profiles have generated concern about the long-term health impact of HAART: however cohort studies evaluating the association of HAART with cardiovascular events have been inconclusive. One study of HIV-infected patients found no increase in the rate of cardiovascular events during more than 8 years of observation. Conversely, a recent study indicated that myocardial infarction incidence was increased with longer HAART duration. Drugs within each class of antiretroviral agents may have different effects on lipid values. Longer follow up and detailed analysis of regimens is needed to further clarify these issues. In the meantime, treatment of lipid abnormalities using the National Cholesterol Education Programme guidelines has been suggested and reported in which many patients required drug therapy for control of lipid abnormalities.

There was a trend towards increasing BMI on ART indicating that the patients gained weight after treatment. No significant change in TSFT was noted in the study as the duration of study was probably short and no PI regimen was studied. Significant increase in waist hip ratio was noted in patients on follow up, however effect of individual drugs on waist hip ratio is difficult to comment upon as combination therapy was given to patients. Significant change in LDL level was noted in patients on follow up. Trend of increasing T.C. level was noted on follow up among patients on ART. Patient on ZLN ART regimen had significant changes in HDL, LDL, TC, and TG favoring dyslipidemia. Similar changes were also noted in patients on ZLE. It was observed that patients having trend towards dyslipidemia also had improving CD4 counts. Whether this was a chance association or not needs to be further investigated. Hence HIV patients on HAART were found to have high prevalence of metabolic syndrome and lipid abnormalities, and they should be regularly screened for the same and appropriately managed. To conclude, larger studies are warranted to compare the effects of switching antiretroviral treatment with those of adding lipid-lowering agents, in order to produce specific recommendations and guidelines for the management of HAART-associated dyslipidemia. We also recommend the implementation of well controlled cohort studies for the evaluation of long term effects of HAART on lipid profiles.

\section{References}

AIDS Res Hum Retroviruses 2012, 28:167278. Pubmed.

Barbaro G: Metabolic and cardiovascular complications of highly active 
antiretroviral therapy for HIV infection: Curr HIV Res 2006, 4:79-85.

Biron A, Bobin-Dubigeon C, Voltaeu C, Piroth L, Perre P, Leport C, et al., Mrtabolic syndromes in French HIVinfected patients: Prevalence and predictive factors after 3 years of antiretroviral therapy.

Grinsztejn B, Luz PM, Pacheco AG, Santos DV, Velsaque L, Moreira RI, et al., 2013. Changing mortality profile among HIV-infected patients in Rio de Janeiro,
Brazil: Shifting from AIDS to nonAIDS related conditions in the HAART era. PLOS one, Vol. 8(4) https://doi.org/10.1371/journal.pone.005 9768

Grover SA, Coupal L, Gilmore N, Mukherjee J. 2005. Impact of dyspipidemia associated with highly active antiretroviral therapy on cardiovascular risk and life expectancy. Am J Car., 95(5):586-91.

\section{How to cite this article:}

Aroop Mohanty, Manish Pandey, A.P. Mohanty and Pratima Gupta. 2018. A Clinical Study of Impact of HIV Infection and HAART on Serum Lipids in People Living in Sikkim. Int.J.Curr.Microbiol.App.Sci. 7(10): 439-443. doi: https://doi.org/10.20546/ijcmas.2018.710.046 